\section{$\underset{\substack{\text { hommes } \\ \text { \& migrations }}}{ }$}

\section{Hommes \& migrations}

Revue française de référence sur les dynamiques

migratoires

1305 | 2014

L'exil chilien en France

\title{
Rendez-vous européen
}

\section{Mustapha Harzoune}

\section{(QpenEdition \\ Journals}

\section{Édition électronique}

URL : http://journals.openedition.org/hommesmigrations/2768

DOI : 10.4000/hommesmigrations.2768

ISSN : 2262-3353

\section{Éditeur}

Musée national de l'histoire de l'immigration

\section{Édition imprimée}

Date de publication : 1 janvier 2014

Pagination : 181-185

ISBN : 978-2919-040261

ISSN : $1142-852 X$

\section{Référence électronique}

Mustapha Harzoune, «Rendez-vous européen », Hommes \& migrations [En ligne], 1305 | 2014, mis en ligne le 11 juillet 2014, consulté le 22 septembre 2020. URL : http://journals.openedition.org/ hommesmigrations/2768; DOI : https://doi.org/10.4000/hommesmigrations.2768 


\title{
RENDEZ-VOUS EUROPÉEN
}

\author{
MUSTAPHA HARZOUNE
}

e 3 octobre 2013, 360 passagers, principalement des Érythréens et des Somaliens, trouvaient la mort au large de Lampedusa. Quelques jours plus tard, deux naufrages, du côté de Malte et de l'Égypte, firent une cinquantaine de victimes, syriennes et palestiniennes. Le 15 novembre, les corps de douze migrants, dont quatre enfants, étaient découverts, noyés, au large de l'île de Lefkada, en mer lonienne. Les drames se succèdent. Jusqu'à quand? Selon l'Office des migrations internationales (OMI), la Méditerranée aurait englouti, depuis 1993, 25 ooo hommes, femmes et enfants. Catherine Wihtol De Wenden, a beau jeu, dans Libération du 27 octobre, d'évoquer les "dysfonctionnements du système migratoire euroméditerranéen”. Dans Jeune Afrique (10 octobre), Olivier Clochard (Migreurop) déclare qu'après le drame à Lampedusa, "la seule décision de la commission de l'Union européenne a été de déclarer une nouvelle augmentation des moyens de Frontex [l'agence de surveillance des frontières européennes] sans faire un bilan sérieux des différentes actions menées jusque-là". Manque d'imagination ou absence de courage politique ? Les dirigeants trembleraient-ils face à la montée en Europe des votes contestataires, anti-européen et anti-immigrés notamment (voir Le Monde du $1^{\text {er }}$ octobre) ? "Le sujet est sensible dans certains États membres. Faire une politique d'immigration coordonnée avec les autres pays européens, c'est prendre le risque d'une montée de l'extrêmedroite" (Franceinfo.fr, 27 octobre). Et le géographe
Michel Lussault interroge :"il est (...) légitime de se demander si la tendance des dirigeants politiques à appréhender l'immigration comme un danger n'est pas la véritable source du problème. En empêchant leur reconnaissance, en les stigmatisant ou en les reléguant dans des banlieues 'ghettoïsantes' notamment, les politiques marginaliseraient d'entrée ces arrivants et ne laisseraient donc que peu de chances à l'immigration de jouer le rôle positif qu'elle peut bien sûr jouer" (rue8glyon.fr, 22 novembre). Cécile Kyenge, ministre italienne de l'Intégration dénonce "I'absurdité" d'une loi italienne qui considère comme des 'suspects' d'immigration clandestine des gens qui fuient des conflits" (Jeune Afrique, 7 octobre). Courage? Imagination?

\section{Quand les chiffrent parlent}

Le 27 octobre, France Info parle des migrants qui "affluent en Méditerranée". Le Figaro du 4 octobre, rapporte qu'“à l'intérieur de l'UE, des responsables s'inquiètent de la paralysie et de l'absence de solidarité 'devant une déferlante qui n'est pas près de se calmer". "Déferlante", "invasion", "pression migratoire"... mais que disent les chiffres ? Le 9 octobre, BFMTV.com fait le point, cartes à l'appui, sur les huit voies d'accès à l'espace Schengen relevées par Frontex et empruntées par les migrants. "Frontex a listé près de 73000 entrées illégales en 2012 dans l'espace Schengen. S'il reste conséquent, le chiffre est très nettement en baisse par rapport 


\section{KIOSQUE}

aux années précédentes : en 2011, 141000 migrants avaient été dénombrés aux mêmes frontières, un pic lié aux 'printemps arabes', et plus de 104000 en 2010. (...) Parmi les nationalités les plus représentées chez les migrants : les Syriens (13\%), les Albanais (12\%) et les Afghans (11\%)." Sur cette baisse, "la plus forte depuis 2005", France info (7 octobre) donne des pourcentages et comparatifs par rapport à 2011. Sur la frontière gréco-turque, avec le renforcement des contrôles et la construction sur 12,5 kilomètres d'un mur de barbelés, "les passages illégaux ont baissé de 35 \% en 2012". En Méditerranée centrale, "la baisse a été de $82 \%$ ". En Méditerranée occidentale, une surveillance renforcée et une "coopération économique avec les pays africains pour freiner le départ de migrants" expliquent "une baisse de $24 \%$ de passages illégaux". En revanche, l'immigration dans les Balkans et en mer Egée est en hausse de $37 \%$ en 2012. Une hausse qui s'explique par l'entrée récente de la Croatie dans l'Union européenne. Dernier secteur où l'immigration clandestine s'est déplacée, la mer Égée et la frontière entre la Turquie et la Bulgarie. La Bulgarie qui a appelé à l'aide l'Union européenne face à l'afflux de réfugiés venus de Syrie. À noter que Jean-François Fiorina sur le cercle. leséchos.fr (28 novembre) citant "diverses associations humanitaires" avance que, pour 2013, "30 000 étrangers" auraient atteint "les seules côtes italiennes depuis le début de l'année, contre 10000 l'an dernier". À suivre donc. Mais, en baisse ou en hausse, ces chiffres montrent que les murs de barbelés et autres drones ne rendront jamais les frontières infranchissables.

\section{L'horizon indépassable de la frontière}

Les raisons de partir, et malheureusement aussi de mourir, sont telles - catastrophes humanitaires, climatiques, environnementales, guerres et persécutions, inégalités de développement... - que des hommes et des femmes tenteront toujours de passer pour vivre. Pourtant, la "tentation de la forteresse" semble être l'unique objet des réflexions et l'horizon indépassable des décideurs. Au point que pour Olivier Bailly, porte-parole de la Commission européenne, "le mouvement de l'Histoire s'est inversé, on en est à chercher comment rétablir les frontières intérieures" traduisant "le chacun pour soi des États européens face aux pressions migratoires à leurs portes" (Républicainlorrain.fr le 25 octobre).

Directmatin.fr du 21 octobre rappelle qu'"entre l'Europe et l'Afrique, bordant l'enclave espagnole de Melilla, s'étire sur onze kilomètres une frontière grillagée de sept mètres de haut, balisée de caméras: une barrière entre deux continents qui ne suffit pas à décourager les assauts de clandestins prêts à tout". Ce "triple grillage" "parait infranchissable" et pourtant, le 17 septembre, 300 migrants se sont lancés à l'assaut de cette frontière métallique. "Cela est un frein. Mais une personne qui a traversé toute l'Afrique, qui a voyagé pendant des mois pour arriver ici, une barrière ne va pas l'arrêter."

Et pourtant. Sur les 259 kilomètres de frontière entre la Bulgarie et la Turquie, "le ministère de I'Intérieur [bulgare] propose l'édification d'une clôture de 30 kilomètres d'une hauteur de 3 mètres (...) dans la zone du mont de Strandja (région d'Elhovo, sud-ouest) par 'où passent environ 85 \%' des immigrants clandestins, la plupart des Syriens" (Le Huffington Post, 16 octobre). Le 20 novembre, le site Levif.be rapporte le point de vue de Paul Collier, professeur d'économie. Directeur du Centre d'étude des économies africaines à l'Université d'Oxford, il "plaide en faveur d'une aide massive de développement aux pays les plus pauvres". Selon lui, il faudrait ériger un mur autour de l'Europe : "Qui souhaitent démolir les murs feraient bien d'instaurer un service de ferries entre l'Afrique $d u$ Nord et l'Europe pour que plus personne ne se noie. Je vous garantis que les ferries seront remplis à 
craquer. (...) Une étude à grande échelle de Gallup révèle que $40 \%$ des habitants des pays pauvres de l'Afrique souhaitent se rendre en Europe. II faut communiquer clairement : ceux qui osent faire la traversée vers Lampedusa seront renvoyés sans état d'âme".

Même logique pour Jacques Delors qui déclare qu' "il faut revoir à la hausse les moyens alloués à l'agence européenne de contrôle aux frontières" (RFI, 11 octobre). En revanche, pour Daniel CohnBendit, "il faut ouvrir l'Europe, donner une possibilité légale de rentrer en Europe, tant qu'on ne le fera pas, on aura les horreurs de Lampedusa". "Les responsables, ce n'est pas l'Europe, ce sont les gouvernements des différents pays (...) qui décident d'une politique aberrante d'immigration qui fait que des gens meurent" (France 3 Midi-Pyrénées repris par rtl.fr,12 octobre).

\section{(In)décisions européennes}

Réunis au Sommet de Bruxelles fin octobre, les 28 chefs d'État et de gouvernement reportaient à décembre des décisions concrètes sur Frontex et à juin 2014 une réforme du droit d'asile. Au moins s'accordaient-ils sur : "L'engagement de donner plus de moyens pour le système de surveillance des frontières de l'UE" autrement dit à l'agence Frontex. Sauf que "l'agence ria pas de moyens propres, et son budget a été réduit (...) de 118 millions dieuros en 2011 à 85 millions cette année" (Le Monde.fr, 9 octobre). Les États membres n'entendent pas se voir dessaisir de la "mission souveraine" de la surveillance des frontières, "les États n'ont pas la même vision de l'immigration", dixit Gil Arias Fernandez, directeur adjoint de Frontex (Le Monde, 27 octobre) et, partant, pas les mêmes politiques d'accueil. "Chaque État entend en effet agir en fonction de ses intérêts propres, de sa dynamique démographique, de ses besoins économiques, de son histoire et de sa situation géogra- phique. Voire des débats politiques et médiatiques du moment, comme on l'a vu en France avec les récentes polémiques sur les Roms" (Jean-François Fiorina, lecercle.les échos.fr, 28 novembre). De là à mettre sur pied une politique migratoire concertée au niveau de l'UE, il y a un grand pas... d'autant plus difficile à faire que la guéguerre entre États se poursuit sur un autre terrain : "Les pays du nord de l'Europe ont refusé de modifier les règles sur l'asile pour aider l'Italie. Comme le Danemark et la Suède, le ministre allemand de l'Intérieur, HansPeter Friedrich, a affirmé que l'accord de Dublin, qui impose aux pays d'arrivée de traiter les demandes d'asile et d'assurer l'hébergement des demandeurs, 'n'a pas à être modifié."

Il a donc fallu attendre décembre pour que Bruxelles propose "un plan d'actions après la tragédie de Lampedusa" qui comprend "un renforcement des patrouilles en mer et dans les airs de Chypre à Gibraltar (...). Frontex a évalué le surcoût de cette présence à 14 millions d'euros par an (...). Un système d'échange d'informations, Eurosur, est opérationnel depuis le 2 décembre pour accélérer les interventions". Mais, poursuit Libération (4 décembre), cette "stratégie ne pourra fonctionner que si des accords sont conclus avec les pays de départ ou de transit des migrants pour les faire intercepter avant qu'ils ne prennent la mer ou lorsqu'ils sont encore dans les eaux territoriales, a expliqué le négociateur. Cela suppose une volonté de coopérer avec l'UE, de l'argent pour former des gardes-côtes et des accords pour la réadmission des clandestins refoulés. L'UE est en pourparlers avancés avec la Tunisie, le Maroc et la Turquie, devenue une plaque-tournante avec le conflit en Syrie". À ce propos, la Turquie a accepté de signer l'accord conclu en juin 2012 pour reprendre les migrants entrés illégalement dans l'UE à partir de son territoire. "En contrepartie, l'UE s'est engagée à garantir une circulation sans visa aux Turcs dans un délai de trois ans et demi" (Lefigaro.fr 16 décembre). La remise en cause réclamée par les pays du Sud 
(Italie, Grèce et Chypre) du règlement de Dublin - qui impose au pays d'entrée d'héberger les migrants, de traiter les demandes d'asile et de renvoyer les clandestins recalés - a été refusée par la majorité des États. "Le problème est pour l'instant contourné par un dédommagement financier. Une enveloppe de 50 millions d'euros est proposée pour aider les pays 'les plus exposés, notamment la Bulgarie, à mettre en place un système d'accueil et d'asile" (Libération, 4 décembre). Jean-François Fiorina sur Lecercle.lesechos.fr (28 novembre), citant Cecilia Malmström, donnait lui d'autres montants: "Pour les seuls fonds consacrés à la gestion des frontières, l'Italie a reçu au cours des deux dernières années 136 millions d'euros, la Grèce a reçu 89 millions d'euros, et Malte a reçu 35 millions d'euros." Le Figaro du 4 octobre : "Tout porte à croire que l'UE en est réduite pour longtemps à une stratégie bancale d'indifférence aux soucis du voisin. Au Nord comme au Sud, les partis populistes ont le vent en poupe et la question de la porosité des frontières, externe mais aussi interne à l'UE dans le cas des Roms, s'impose déjà dans la campagne pour les européennes du printemps 2014." Jean-François Fiorina ajoute : "Nous touchons du doigt les limites d'une 'construction européenne' qui ne s'assume pas politiquement et juridiquement, tout en contribuant à délégitimer idéologiquement les leviers d'intervention strictement étatiques. Or le sujet est grave, donc d'essence politique, et pas seulement humanitaire" (Lecercle.lesechos.fr, 28 novembre).

\section{Envisager une autre politique}

Dans Jeune Afrique (10 octobre), Olivier Clochard, chargé de recherche au CNRS et président du réseau Migreurop, dénonce I'“hypocrisie" des Européens, l'"attitude criminelle" d'une "Europe assassine" et cette "politique du 'tout répressif". "En 2005, environ 1000 personnes sont mortes en tentant de traverser la Méditerranée. Deux ans plus tard, en 2007, l'agence a obtenu des moyens supplémentaires (...). Nombre de morts : environ 1800. En 2011, le Conseil européen a décidé d'encore augmenter les capacités de Frontex. Nous sommes passés à plus de 2000 décès. Plus ils mettent de moyens, plus les choses empirent." "Or, si on veut diminuer le nombre de morts aux frontières, [il faudrait] arrêter le 'tout sécuritaire'. Depuis plus de dix ans, nous sommes dans cette logique et nous voyons les mauvais résultats que cela a donnés." Pour François Crépeau, rapporteur spécial de l'ONU sur la protection des migrants, en verrouillant leurs frontières les pays européens ne font en réalité que "donner plus de pouvoir" aux passeurs et aux trafiquants d'êtres humains. II dénonce une "paranoïa" entretenue par la classe politique. "On aurait pu éviter ces morts. Traiter l'immigration clandestine uniquement par des mesures répressives est susceptible de provoquer ces tragédies. L'immigration clandestine n'est pas un crime contre les personnes ou les biens ni une menace pour la sécurité" (Le Monde.fr, 4 octobre). Et d'ajouter, qu'“il faut développer les possibilités d'immigration légale". Tel est aussi le point de vue d'Olivier Clochard: "Nous pourrions aussi favoriser une meilleure mobilité entre les pays des deux rives de la Méditerranée."

Une option d'autant plus envisageable qu'aux politiques de fermeture répondent des stratégies toujours nouvelles d'adaptation et de contournement. Ainsi, dans un entretien donné au site Atlantico.fr, le 5 octobre, Cris Beauchemin cite "de récents travaux menés sur les migrants subsahariens (dans le cadre du projet 'Migrations entre l'Afrique et l'Europe' : http://mafeproject.site.ined.fr/) [qui] montrent que les trajectoires des migrants sont très mouvantes". "C'est un fait quasi universel : les fermetures de frontières ne bloquent pas radicalement les migrants, elles les forcent à adapter leurs trajectoires, à contourner les lieux les plus contrôlés et, aussi, à prendre plus de risques." II rappelle que 
la politique de contrôle "mériterait d'être évaluée sérieusement parce que son efficacité est douteuse (les tentatives de migrations perdurent) et parce qu'elle comporte de nombreux effets pervers (...) [comme celui] de réduire les migrations de retour alors même que les pays européens souhaiteraient les encourager". Dans le même entretien, Jacques Barou souligne que si l'Union européenne a créé l'agence Frontex, elle "a eu une meilleure initiative en faisant de l'information dans les pays de départ pour prévenir les migrations à risque. (...) Beaucoup ont pu prendre conscience du danger qu'ils allaient courir pour des gains peu évidents".

Radical, Olivier Pastré écrit que "I'Europe a presque tout faux" (Libération, 24 novembre) : "L'immigration est une chance. Nos principaux concurrents à l'échelle mondiale l'ont bien compris. Retrouvons juste un peu de cohérence. Soyons plus inflexibles que nous le sommes sur l'immigration clandestine et, au contraire, soyons plus offensifs et plus imaginatifs sur l'accueil et l'intégration des populations immigrées dont nous avons fondamentalement besoin. Soyons égoïstes et, pour cela, soyons plus hospitaliers. Vite. Car le temps de la mondialisation presse."

Last but not least, le 14 novembre, le site Slateafrique.com pointe "La grande part de responsabilité des dirigeants africains dans les naufrages à répétition. Le manque de politiques de développement cohérentes et le peu de considération à l'égard de leurs peuples sont autant de raisons qui expliquent cette périlleuse fuite des Africains vers l'Europe." Toute chose égale par ailleurs, puisque les Africains ne représentent qu'une faible partie de l'immigration clandestine en Europe.

Catherine Wihtol de Wenden dans Libération du 27 octobre résume : "De nombreux flux, de créations d'entreprises, de commerce, d'échanges, de mariages, de liens transnationaux traversent la Méditerranée malgré la sévérité des barrières opposées à la mobilité dans la région." Elle rappelle qu'“une complémentarité existe entre les marchés du travail européens et leur profil démographique et la disponibilité d'une main-d'ouvre qualifiée et non qualifiée sur la rive sud". Elle pointe les ambiguïtés d'une politique de fermeture des frontières obligée de s'accommoder "de nombreuses pratiques et bricolages institutionnels et factuels, passages clandestins, double nationalité, réseaux transnationaux familiaux, économiques et médiatiques, régularisations, accords bilatéraux entre pays de la rive nord et de la rive sud qui tantôt en atténuent la rigueur, tantôt marchandent les reconduites aux frontières contre des politiques de développement ou des titres de séjour pour les plus qualifiés, ou encore soulignent l'absurdité d'une clôture dans un lieu aussi riche d'échange".

Partant, elle aussi avance l'hypothèse qu' "une entrouverture, ne serait-ce que minime, des frontières dans la région [euroméditerranéenne] (...) serait une source de richesse accrue". À I'heure où "la migration est un phénomène structurel des sociétés" et que "le monde rencontre le monde", la politique de fermeture des frontières "est porteuse d'insécurité, de terrorisme et de fanatismes en tous genres, tant au Sud qu'au Nord, alors que c'est au nom de la sécurité que l'on cherche à fermer les frontières".

Pour Dominique Moïsi, professeur au King's College de Londres, "l'attrait de l'Europe pour les réfugiés de l'autre côté de la Méditerranée ne va pas disparaitre comme par un coup de baguette magique. L'inégalité des situations demeure trop grande. De la même manière la montée des partis populistes européens semble (...) inévitable. La responsabilité des gouvernements européens est d'éviter toute dérive politicienne. On ne peut ni répondre aux arguments des populistes en fermant nos frontières, ni se contenter de rester sur le registre de l'indignation humanitaire. Ce qui est déjà difficile au niveau national peut devenir tout simplement impossible à vingt-huit. Mais ce serait suicidaire pour l'Europe de rater ce rendez-vous" (Les Échos, 28 octobre). 